\title{
A multi-centre early evaluation of the effectiveness of workshop teaching to improve the confidence of UK and Irish dental students when addressing patient mental health
}

\author{
Emma Elliott, ${ }^{* 1}$ Sathyam Sharma, ${ }^{1}$ Alya Omar, ${ }^{2}$ Dominic Hurst, ${ }^{3}$ Catherine Marshall, ${ }^{4}$ Alex Blair, ${ }^{5}$ Alison McCullagh, ${ }^{6}$ \\ Anusha Chopra, ${ }^{7}$ Aurelia Claudia, ${ }^{8}$ Gopi Patel, ${ }^{7}$ Harriet Beaty, ${ }_{1}^{8}$ Shahd Busnaina ${ }^{6}$ and Varsha La $^{6}$
}

\section{Key points}

Dental students across the UK and Ireland selfreport low confidence when addressing patient mental health in the dental clinic, especially when addressing undiagnosed psychiatric conditions.
Interactive workshop teaching is an effective method to significantly improve the confidence of dental students when addressing patient mental health.
This study recommends the implementation of interactive workshop teaching within BDS curricula to ensure graduates can 'identify, explain and manage the impact of medical and psychological conditions', as recommended by the GDC.

\begin{abstract}
Background Dental practitioners can have low confidence when addressing patient mental health as part of wider patient management. This is increasingly relevant due to the rising prevalence of mental health conditions and the relationship that can exist between mental and oral health. Interactive workshop teaching on patient mental health may enhance the confidence of dental students when addressing mental health conditions in patients. This study trialled workshop teaching as an educational intervention in five UK and Irish dental schools.
\end{abstract}

Methods A quantitative, scenario-based confidence survey to further establish the need for intervention, followed by delivery of a workshop intervention to volunteer participants. Pre- and post-workshop surveys were used to assess the effectiveness of the workshop.

Results Survey data showed low confidence among dental students when addressing patient mental health. Workshop intervention improved dental student confidence on average from 2.3-3.7 on a five-point scale, with less than $0.5 \%$ likelihood that reported changes in confidence were due to chance.

Discussion Low confidence of dental students addressing patient mental health scenarios echoed wider literature findings surrounding dental clinicians' ability to address patient mental health. This further demonstrated the need for educational intervention, with workshop effectiveness demonstrated within this paper.

Conclusion Workshop teaching is an effective way to enhance dental student confidence when addressing patient mental health and should be considered for implementation in the Bachelor of Dental Surgery curriculum.

'Undergraduate Student, Institute of Dentistry, Barts and The London School of Medicine and Dentistry Dental Hospital, Turner Street, London, E1 2AD, UK; ${ }^{2}$ Former Undergraduate Student, Institute of Dentistry, Barts and The London School of Medicine and Dentistry Dental Hospital, Turner Street, London, E1 2AD, UK; ${ }^{3}$ Queen Mary University of London, Institute of Dentistry, Barts and The London School of Medicine and Dentistry Dental Hospital, Turner Street, London, E1 2AD, UK; ${ }^{4}$ Centre for Psychiatry, Barts and the London School of Medicine and Dentistry, Queen Mary University of London, London, EC1M 6BQ, UK: ${ }^{5}$ Undergraduate Student, Dundee Dental Hospital and School, 2 Park Place, Dundee, DD1 4HR, UK; ${ }^{6}$ Undergraduate Student, Dublin Dental University Hospital, Lincoln Place, Dublin, D02 F859, Ireland; ${ }^{7}$ Undergraduate Student, King's College Dental Institute, Guy's Hospital, Great Maze Pond, London, SE1 9RT, UK; ' Undergraduate Student, Newcastle School of Dental Sciences, Framlington Place,

Newcastle Upon Tyne, NE2 4BW, UK.

*Correspondence to: Emma Elliott

Email address: emmaelliottdental@hotmail.com

Refereed Paper

Accepted 8 February 2021

https://doi.org/10.1038/s41415-021-3613-8

\section{Background}

Psychiatric disorders are becoming more common among the UK population. One in four people will experience a mental health problem in any given year, ${ }^{1}$ and in 2016 , $20 \%$ of men and $30 \%$ of women had been diagnosed with a mental health condition. ${ }^{2}$ NHS adult psychiatric morbidity surveys have additionally reported an increase in prevalence of common mental disorders since 1993. ${ }^{1}$ For dental practitioners, there is a need to be aware of such population changes and to be able to effectively manage our patient base.

Psychiatric disorders are often associated with low self-esteem, self-neglect, increased sugar intake, substance abuse and irregular dental attendance. ${ }^{3}$ Consequently, oral symptoms may be early or the sole identifiable physical manifestations of underlying psychiatric disorders. ${ }^{4}$ The General Dental Council (GDC) states that dentists should be able to 'identify, explain and manage the impact of medical and psychological conditions' as part of patient management. ${ }^{5}$ From the perspective of both the GDC and holistic patient care, it is within the dental scope of practice to have an awareness of and to integrate psychiatry within dentistry, in order to minimise patients 'falling through the gaps' of psychiatric services. ${ }^{6}$

Previous studies have highlighted the low confidence dentists feel when addressing patients who have psychiatric conditions, 
where $46 \%$ of surveyed UK dentists did not refer patients with mental health conditions and if they did make a referral to a specialist dentist it was for a physical manifestation, rather than psychological. ${ }^{7}$ Often, dentists may have preconceived notions about how a patient might react to a discussion linking their oral health with their mental health, with $33 \%$ of surveyed dentists assuming a patient would be insulted by such a conversation. ${ }^{8}$ These assumptions can come from the lack of formal education received in this area, with the same study outlining that knowledge about psychiatric disorders and specifically eating disorders often came from media or personal experience, not dental school. ${ }^{8}$

Currently, UK dental students report low confidence when addressing patient mental health and it is likely that this low confidence is due to the limited focus within the Bachelor of Dental Surgery (BDS) curriculum.' Addressing patient mental health refers to 'the ability to understand the impact of mental health on dental treatment, ask further questions in order to appropriately gauge a medical history, make psychiatric referrals or make recommendations to visit a GP'. ${ }^{9}$ Consequently, workshops and communication skills teaching have been identified by dental students as methods to enhance their confidence and ability to address psychiatric conditions among patients. ${ }^{9}$

Cochrane Reviews have established workshop teaching as a method that is able to introduce small to moderate changes in professional outcomes, highlighting that interactive education is additionally more effective than didactic learning. ${ }^{10}$ Further research has also demonstrated that in continuing medical education (CME), 'formal interactive CME interventions are effective in changing physician performance. ${ }^{11}$ As a result of dental student recommendation, CME research and Cochrane evidence, the core researchers (EE, AO, SS, CM and $\mathrm{DH}$ ) developed an interactive, communication skills-based workshop, designed to be delivered to clinical years of the BDS curriculum. This study planned to further investigate UK and Irish dental students' confidence when

Table 1 Dental schools involved in the study and the appointed researchers responsible for conducting the research within each school

\begin{tabular}{|c|c|}
\hline Dental school & Appointed researchers \\
\hline $\begin{array}{l}\text { Barts and the London Dental School, Queen Mary } \\
\text { University of London }\end{array}$ & Emma Elliott, ${ }^{*}$ Sathyam Sharma* and Alya Omar* \\
\hline King's College London & Gopi Patel and Anusha Chopra \\
\hline Trinity College Dublin, University of Dublin & Varsha Lal, Alison McCullagh and Shahd Busnaina \\
\hline Newcastle Dental School & Harriet Beaty and Aurelia Claudia \\
\hline Dundee Dental School & Alex Blair \\
\hline \multicolumn{2}{|c|}{$\begin{array}{l}\text { "Key: } \\
\text { "= Student researchers at Barts and the London Dental School (EE, SS and AO) were responsible for the design and } \\
\text { implementation of the overall study, alongside Dr Dominic Hurst and Dr Catherine Marshall." }\end{array}$} \\
\hline
\end{tabular}

Table 2 Survey scenarios

\begin{tabular}{|c|c|}
\hline $\begin{array}{l}\text { Mental health } \\
\text { condition }\end{array}$ & Scenario and question \\
\hline Depression & $\begin{array}{l}\text { You are screening a new patient, Mr Frederick, a 50-year-old man. As part of the medical } \\
\text { history, you ask if he suffers from any psychiatric disorders. After hesitating, he states he } \\
\text { suffers from depression. The form requires that you 'give details' about his depression. How } \\
\text { confident would you feel asking Mr Frederick further questions about his depression? }\end{array}$ \\
\hline Eating disorders & $\begin{array}{l}\text { Ms Patel is a 20-year-old patient. You notice she is severely underweight and has a history } \\
\text { of severe caries, so ask her to fill out a diet sheet. She later returns the diet sheet, which } \\
\text { seems heavily exaggerated and does not seem to correspond to her frame. You suspect } \\
\text { the patient may be hiding an eating disorder. How confident would you feel asking Ms } \\
\text { Patel questions about a potential eating disorder? }\end{array}$ \\
\hline Anxiety & $\begin{array}{l}\text { You are screening Ms Angelou, a } 40 \text {-year-old woman. She declares she has been } \\
\text { recently diagnosed with generalised anxiety disorder. How confident would you feel in } \\
\text { appropriately managing Ms Angelou's dental care? }\end{array}$ \\
\hline Schizophrenia & $\begin{array}{l}\text { You are treating Mr Chand, a 34-year-old man. He claims to have no medical history; } \\
\text { however, he reveals he is taking medication which you recognise to be prescription anti- } \\
\text { psychotic medication. How confident would you feel returning to the psychiatric disorders } \\
\text { section of the medical history and asking further questions? }\end{array}$ \\
\hline
\end{tabular}

addressing patient mental health and then deliver intervention-based workshop teaching to address the issue.

\section{Methodology}

\section{Study design}

The core researchers developed an approach of a quantitative, scenario-based confidence survey to further establish the need for intervention, followed by delivery of an educational workshop intervention to volunteer participants. Pre- and post-workshop surveys were used to assess the effectiveness of the workshop. The study was implemented across five dental schools, with each school having an interviewed and appointed team of student researchers who would conduct the study (Table 1).

\section{Scenario-based confidence survey}

The scenario-based confidence survey involved four fictional scenarios where patient mental health presented within the dental clinic (Table 2). These scenarios were generated by the core researchers based upon common mental health disorders among the UK population. Each scenario asked the respondent to rate how they would feel asking further questions or how confident they were in their ability to manage the patient. Participants rated themselves on a scale of 1 ('no confidence') to 10 ('absolute confidence') for each scenario.

This survey was intended to identify the confidence felt by UK and Irish dental students as a population and to potentially further evidence the need for the workshop intervention. The scenario-based confidence survey was conducted in each dental school before the workshop was delivered. The workshop teaching was to take place regardless of the survey results and the survey did not influence the design of the workshop, which had been developed before the study began and was based on previous work in one UK dental school. ${ }^{9}$

This survey was created using SurveyMonkey or Google Forms and was emailed as an online link to all dental students in clinical year groups of the BDS degree at each institute. Clinical year groups were defined as year groups where dental students had patientfacing contact; typically, years three, four and five. The survey was not assessed for validity beyond face validity by the core researchers and the results were described using simple descriptive statistics. 
Fig. 1 Structure of workshop delivery and content
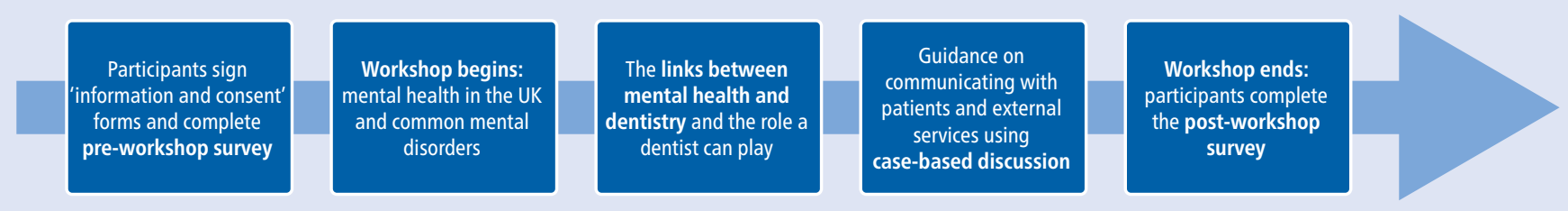

\section{The workshop intervention}

An educational workshop designed to cover psychiatry within dentistry was created by the core researchers, including Dr Catherine Marshall, a clinical lecturer in psychiatry. The workshop was constructed to cover key topics outlined in Figure 1 and was intended to last between 1.5-2 hours. Case-based discussion was considered to be a focal point at the end of the workshop to link together the discussed concepts and to allow students to apply this knowledge within a small, group setting. In order to promote student engagement and open conversation with these case discussions the workshop was limited to 20 participants.

The workshop was delivered within each institute by the student researcher(s) and a mental health specialist local to each dental school. Volunteer participants from clinical year groups were invited to participate.

\section{Pre- and post-workshop surveys}

Workshop participants completed pre- and postworkshop surveys, which contained a mix of quantitative and qualitative questions (Table 3). The core researchers developed these questions and they were not validated beyond face validity. These surveys were used to assess average participant progress following the workshop and judge the effectiveness of the intervention. Quantitative aspects of the surveys were analysed using statistical analysis, applying a paired t-test to the confidence scale questions that appeared in both the pre- and post-workshop surveys.

\section{Participants and setting}

Research participants were students from the clinical years of the BDS degree at five different dental schools. Convenience and volunteer sampling ${ }^{12}$ was used to recruit participants for the workshop. It is important to note that the workshop was an extracurricular activity and thus was not officially timetabled, limiting participant availability.

Students were invited to participate in the workshop by email and face-to-face. Participant information and consent forms were distributed before each workshop. ${ }^{12}$

Table 3 Pre- and post-workshop survey questions. Questions overlapped to monitor changes in student confidence before and after the workshop

\begin{tabular}{l|l} 
Pre-workshop survey questions & Post-workshop survey questions \\
\hline What year of BDS are you in? & \\
\hline $\begin{array}{l}\text { Do you study BDS as an undergraduate or } \\
\text { a postgraduate? }\end{array}$ & \\
\hline $\begin{array}{l}\text { What is your age? } \\
\text { Tick box: }<25, \leq 25-30,31 \text { or over, rather } \\
\text { not say }\end{array}$ & \\
\hline
\end{tabular}

Dental signs and symptoms present as a consequence of mental health problems. How confident do you feel about recognising these?

Scale of $1-5$ from'not confident' (1) to 'very confident' (5)

How confident would you feel talking to a patient about their mental health?

Scale of 1-5 from 'not confident' (1) to 'very confident' (5)

If a patient presented with a mental health problem, how confident would you feel in your ability to address this if necessary?

Scale of 1-5 from 'not confident' (1) to 'very confident' (5)

If you decided to refer a patient for further help, would you know what options are available to you? If so, please outline these options.

Checkbox of 'yes' or 'no'. Space then provided to outline the options

Feedback: 'I found today's workshop useful'. Five-point scale from 'strongly disagree' (1) to 'strongly agree' (5) and option to explain why this rating was given

Feedback: 'I enjoyed today's workshop'. Five-point scale from 'strongly disagree' (1) to 'strongly agree' (5) and option to explain why this rating was given

Feedback: how would you rate the style of information delivery, the case discussions and the workshop timings? Five-point scale from 'very poor' (1) to 'very good' (5) for each sub-section

Feedback: lastly, do you have any suggestions for improvements to the workshop? Space provided

\section{Ethics}

Ethical approval was granted independently by each of the five institutional ethics departments: QMREC2321a (Queen Mary University of London), DSREC122019/01 (Trinity College Dublin), MRSU-19/2015011 (King's College London), UoD $\backslash S D E N \backslash$ UG\2019030 (Dundee) and 1867/848/2020 (Newcastle).

\section{Results}

\section{Quantitative results}

Scenario-based confidence survey

In total, 1,285 dental students from five UK and Irish dental schools were sent the survey, with 225 respondents, giving an average response rate of $18.8 \%$. The results were collated and presented as average dental student confidence per question in Figure 2. Average self-reported student confidence was low, averaging 4.86/10 across all four questions. Confidence was lowest in scenario two, where an undiagnosed eating disorder was directly impacting dental treatment.

\section{Workshop participant demographics}

Overall, 63 dental students participated in the workshop teaching. The lowest attendance for an individual workshop was six participants and the highest attendance was 20 participants, with an average attendance of 12.6 participants per workshop. The majority of participants were in their fourth year of BDS (49.2\%), with $28.8 \%$ in their fifth year and $22 \%$ in their third. It was anticipated that 
third-year students might negatively affect the averages; however, on analysis the average 'pre-workshop survey' confidence scale of those in third year was 2.25 and of those in fifth year 2.18.

Demographic data on workshop participants were limited to age range and whether they were graduate-entry or undergraduate-entry to the BDS programme (Figures 3 and 4).

Two workshops were delivered in person as originally intended. Due to the COVID19 pandemic, three dental schools delivered the workshop virtually via Zoom or a similar service (Table 4). All workshops were co-delivered by the student researcher(s) and a mental health specialist local to that school. At Trinity College Dublin, the student researcher held a previous degree in psychology and so fulfilled both roles, alongside a local dentist with a special interest in mental health.

\section{Pre-workshop and post-workshop survey comparison}

Results were analysed using a paired t-test for the following three questions:

1. Dental signs and symptoms present as a consequence of mental health problems. How confident do you feel about recognising these?

2. How confident would you feel talking to a patient about their mental health?

3. If a patient presented with a mental health problem, how confident would you feel in your ability to address this if necessary?

Workshop participants were asked these three questions in the pre- and post-workshop survey to study how the workshop intervention influenced their confidence. These questions were felt to represent major teaching components of the workshop. Each question was answered on a scale of 1 ('not confident') to 5 ('very confident'). Across all questions, the average increase in confidence was from 2.33.7. For each question and data set, the $\mathrm{p}$ value was $<0.005$, hence changes in confidence are less than $0.5 \%$ likely to be due to chance. Figure 5 outlines the changes in confidence pre- and post-workshop, with the $\mathrm{Y}$ axis representing the scale of 1-5 from 'not confident' (1) to 'very confident' (5).

Pre-workshop, only $7.6 \%$ of participants knew where they could refer a patient for further help regarding their mental health and were able to outline what they could do. Post workshop, $92.5 \%$ of participants were aware of

\section{Fig 2 UK and Irish dental student confidence ratings per question}



Fig 3 Age distribution of participants attending the workshops

Fig 4 Ratio of 'undergraduate-entry to BDS' to 'graduate-entry to BDS' workshop attendance

Table 4 Schools which ran in-person workshops and schools which ran the workshop virtually

\begin{tabular}{l|l} 
In-person workshop (pre-pandemic) & Virtual workshop (during pandemic) \\
\hline $\begin{array}{l}\text { Barts and the London Dental School, Queen Mary } \\
\text { University of London }\end{array}$ & Newcastle \\
\hline Trinity College Dublin, University of Dublin & King's College London \\
\hline & Dundee \\
\hline
\end{tabular}

where they could refer such patients and what services they could communicate with.

\section{Feedback and qualitative results}

In total, $57.4 \%$ of workshop participants strongly agreed with the statement 'I found today's workshop useful' and the remaining participants either agreed with the statement $(39.3 \%)$ or were neutral to the statement (3.3\%). Selected participant comments about the usefulness of the workshop are highlighted below:

- 'Great conversations and enlightening communication skills which can be put into practice with patients'
- 'The most useful aspect of the workshop for me was going through the different options for referral that are available to us as dentists. I feel more confident about how to bring up mental health with my patients.'

In total, 58.3\% of workshop participants strongly agreed with the statement 'I enjoyed today's workshop' and the remaining participants either agreed with the statement (36.7\%) or were neutral to/disagreed with the statement (5\%). Selected participant comments about what they enjoyed most are highlighted below: 
Fig 5 Average pre- and post-workshop participant confidence with confidence intervals for questions one, two and three

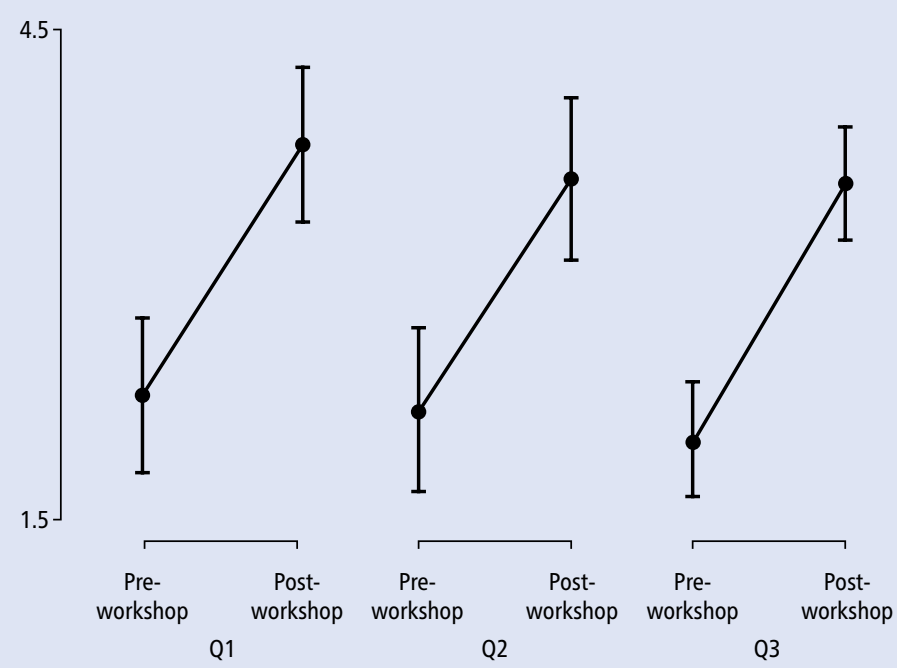

- 'The interactive nature, particularly [case discussion]. Easy to understand the concepts presented'

- 'So insightful, really enjoyed it and it has really made me much more confident to approach the topic of mental health with patients. Such relevant information.'

Style of information delivery and the inclusion of case discussions were rated either very good or good by approximately $95 \%$ of participants, with the remaining $5 \%$ being neutral. When it came to workshop timings, $83.3 \%$ of participants rated the timings as very good or good, $15 \%$ were neutral and $1.7 \%$ rated the timings as poor. Finally, participants were asked if they had any suggestions for improvements to the workshop. Selected suggestions that best represented the feedback are highlighted below:

- 'Handout summary of key points and guidelines summary of how to manage mental health issues'

- 'Clinical photographic examples of some of the oral manifestations of mental illness, as this would be easier to visualise what we were looking for.'

\section{Discussion}

The general low confidence of dental students across the baseline confidence survey scenarios reflected wider literature findings investigating the ability of dentists to address patient mental health, suggesting that there has been limited focus on enhancing teaching in this field. ${ }^{7,8}$ This further demonstrated the need for effective, formalised education of psychiatry within dentistry. Self-reported confidence was lowest when addressing the eating disorder scenario in the confidence survey. This low confidence likely related more to the context of the scenario, rather than the topic of eating disorders themselves. The scenario linked severe caries with the patient's underlying psychological health and as such, demanded action and awareness of pathways available.

As indicated by a previous Cochrane review ${ }^{10}$ and CME research, ${ }^{11}$ an interactive, educational workshop can be an effective educational tool. In this study, student confidence increased as a result of the workshop in three target areas: confidence at recognising dental presentations that can relate to mental health, confidence when talking to their patients about mental health and confidence in their ability to generally address patient psychiatric disorders. It has been highlighted that, in medical undergraduate programmes, in-person psychiatry lectures are preferred as opposed to video recordings, suggesting interactivity is a component to student learning. ${ }^{13}$ Additionally, medical students believe their learning would be enhanced when focusing on commonly encountered psychiatric scenarios. ${ }^{14}$ Our workshop was able to encompass these elements and evidence the success of such a 'student-focused' intervention design.

The split between virtual workshop teaching in three schools and in-person workshop teaching at two schools had no negative impact on the overall average changes in participant confidence. This suggests that online educational tools can be equally effective and indicates success of virtual education during the
COVID-19 pandemic. However, our data were too small to make a conclusive statement on the comparative effectiveness of the two delivery methods and further investigation is warranted.

Student feedback was overwhelmingly positive. The workshop was considered useful and enjoyable, with feedback asking for more fictional, case-based discussion and for handouts or guideline summaries. The development of guidelines for dental clinicians addressing patient mental health has long been discussed, with their development proposed in a 2001 paper. $^{7}$ In 2000, the British Society for Disability and Oral Health created 'Oral healthcare for people with mental health problems: guidelines and recommendations, ${ }^{3}$ which primarily focused on the dental disease presentation of those with mental health disorders and barriers to accessing dental care.

Although pertinent, these guidelines do not provide a framework of action for a dental practitioner concerned about a patient's mental health, making only a small reference to which services should be liaising with each other to overcome barriers to accessing dental care. There is still a need for guideline development for dental clinicians to be able to understand how to liaise externally when the patient is previously unknown to psychiatric services. In addition to supporting education in this area by evidencing the positive impact workshop teaching can have, we also highlight the need for further guideline development.

This study demonstrates that there is a need for education of psychiatry within dentistry and that students are receptive and engaged with teaching in this area, if they are able to attend. In accordance with the GDC statement 'dental graduates should be able to identify, explain and manage the impact of medical and psychological conditions, ${ }^{5}$ this study recommends including teaching on psychiatry within dentistry as part of the BDS curricula. The core researchers intend to develop an interactive, e-module workshop that can be delivered within dental schools. Under the COVID-19 pandemic, we feel that this is the most sustainable way to officially introduce such teaching into the curriculum.

\section{Limitations}

The scenario-based survey and pre- and postworkshop surveys were not validated beyond face validity. Given the widespread nature of the five dental schools involved in the study, we feel that the results are generalisable to the UK and Irish dental student population. 
We collected limited demographics data and cannot comment on the diversity of the students who participated in the study. Additionally, we cannot comment on how selfreported confidence would translate into real clinical behaviour. While we feel our results could be applicable to other oral healthcare professionals, our study did not focus on their curricula or their confidence regarding patient mental health.

\section{Conclusion}

In the UK and Ireland, dental students selfreport low confidence when addressing fictionalised patient mental health scenarios. Interactive, educational, workshop-based teaching, including fictionalised case discussions and co-delivery alongside a mental health specialist, was an effective tool for improving dental students' confidence with patient mental health. We recommend that this should be considered for implementation into undergraduate dental student curricula and intend to act on this with the creation of an interactive e-module workshop for use in dental schools.
Acknowledgements

We would like to acknowledge the contribution of Dr Lina Zariddin, Dr Richard Lee Kin, Dr Tiago Costa and Dr Mourad Wahba for their help in delivering the workshops across Dundee, Trinity College Dublin and Newcastle.

\section{Ethics declaration}

The authors declare no conflicts of interest.

\section{References}

1. McManus $S$, Bebbington $P$, Jenkins $R$, Brugha T. Mental health and wellbeing in England: Adult Psychiatric Morbidity Survey 2014. NHS Digital. 2016. Available at https://digital.nhs.uk/data-and-information/ publications/statistical/adult-psychiatric-morbiditysurvey/adult-psychiatric-morbidity-survey-survey-ofmental-health-and-wellbeing-england-2014 (accessed October 2021).

2. Mental Health Foundation. Fundamental Facts About Mental Health 2016. 2016. Available at https://www. mentalhealth.org.uk/publications/fundamental-factsabout-mental-health-2016 (accessed October 2021).

3. BSDH Working Group. Oral Health Care for People with Mental Health Problems: Guidelines and Recommendations. 2000. Available at https://www. bsdh.org/index.php/retracted-guidelines (accessed October 2021).

4. Kisely S. No Mental Health without Oral Health. Can J Psychiatry 2016; 61: 277-282.

5. General Dental Council. Preparing for Practice: Dental Team Learning Outcomes for Registration (2015 Revised Edition). 2015. Available at https:// www.gdc-uk.org/education-cpd/quality-assurance/ learning-outcomes-review-process (accessed October 2021).

6. Mind. Mental Health in Primary Care: A briefing for Clinical Commissioning Groups June 2016. 2016. Available at https://www.mind.org.uk/about-us/ourpolicy-work/reports-and-guides/primary-care-reports/ (accessed October 2021)

7. Lloyd-Williams F, Dowrick C, Hillon D, Humphris G, Moulding $\mathrm{G}$, Ireland R. A preliminary communication on whether general dental practitioners have a role in identifying dental patients with mental health problems. Br Dent J 2001; 191: 625-629.

8. Johansonn A K, Nohlert E, Johansonn A, Norring C, Tegelberg Å., 2009. Dentists and eating disorders knowledge, attitudes, management and experience. Swed Dent J 2009; 33: 1-9.

9. Elliott E, Sharma S, Omar A, Hurst D. How confidently do students address patients with psychiatric conditions in the dental clinic? A service evaluation in a UK dental school. Br Dent J 2020; 228: 376-380.

10. Forsetlund L, Bjørndal A, Rashidian et al. Continuing education meetings and workshops: effects on professional practice and health care outcomes. Cochrane Database Syst Rev 2009; DOI: 10.1002/14651858.CD003030.pub2.

11. Holm H. Review: interactive, but not didactic, continuing medical education is effective in changing physician performance. Evid-Based Med 2000; 5: 64.

12. Teddlie C, Yu F. Mixed methods sampling: A typology with examples. J Mix Methods Res 2007; 1: 77-100.

13. Petrie K, Trollor J, Dean K, Harvey S. Medical students' preferences regarding Psychiatry teaching: a comparison of different lecture delivery methods. MedEdPublish 2019; D0I: 10.15694/ mep.2019.000163.1.

14. Oakley C, Oyebode F. Medical students' views about an undergraduate curriculum in psychiatry before and after clinical placements. BMC Med Edu. 2008; DOI: 10.1186/1472-6920-8-26. 